\title{
Open Abdominal Aortic Aneurysm Repair: Detection of Delayed Aneurysmal Complications
}

Perera KG*, Wong Ed and Yasir H

Eastern Health, Nil financial support, 8 Arnold St., Box Hill, Victoria 3128, Australia

\begin{abstract}
Purpose: Up to $65 \%$ of open abdominal aortic aneurysm (AAA) repairs develop para-anastomotic pseudoaneurysms; $14 \%$ of these requiring surgery. A further $30 \%$ may develop metachronous common iliac artery $(\mathrm{CIA})$ aneurysms with up to $15 \%$ being surgically significant. Conventional management is to perform a surveillance scan 5 years post-operatively, yet many of these patients are lost to follow-up. This study evaluates the incidence of such aneurysms in our community and will determine appropriate follow-up practice.
\end{abstract}

Methodology: Patients having undergone open AAA repair at Eastern Health at least 5-years prior to the study date were identified. Deceased patients and those requiring high level nursing care were excluded. Eligible cases were reviewed in clinic and offered CT evaluation where there was no recent scan available.

Results: 171 operations were identified between 2003- 2008. 90 (53\%) patients were confirmed as deceased; with a further $45(26 \%)$ unable to be contacted. Of the 36 eligible patients, $18(50 \%)$ agreed to participate in clinical assessment with only 9 subsequently attending a review clinic; one of whom passed away prior to scanning. $5(56 \%)$ were found to have developed a para-anastomotic aneurysm and $2(22 \%)$ had CIA aneurysms.

Conclusions: Despite the limited response rate, the incidence of both para-anastomotic pseudoaneurysms and $\mathrm{CIA}$ aneurysms in our experience is significant to warrant closer surveillance. Routine follow-up with imaging should be considered for the detection of delayed aneurysm development.

Keywords: Abdominal aortic aneurysm; Follow-up; Surveillance; Pseudo aneurysm

\section{Introduction}

Abdominal aortic aneurysm (AAA) is a relatively common vascular condition that affects the aortic wall [1]. With risk increasing in direct proportion to age, the incidence and prevalence of AAAs is set to rise. Up to $65 \%$ of open AAA repairs develop para-anastomotic pseudoaneurysms; $14 \%$ of these requiring surgery. A further $30 \%$ may develop metachronous common iliac artery (CIA) aneurysms with up to $15 \%$ being surgically significant [2]. Patients surgically treated with open aneurysm repair have a risk of delayed development of metachronous peripheral aneurysms and paraanastomotic pseudoaneurysms.

Conventional management is to perform a surveillance scan 5 years post-operatively, yet many of these patients are lost to follow-up. This study evaluates the incidence of such aneurysms in our community and will determine appropriate follow-up practice following open aneurysm repair.

Prior studies have used follow-up ultrasound and computed tomography (CT) scanning for investigation of metachronous aneurysms in patients having undergone AAA repair. Hallett et al. performed a combination of such imaging on 140 patients to discover a proximal aortic false aneurysm prevalence of $1 \%$ over a 36 -year period [3]. Based in Toronto, Kalman et al. solely utilised CT scanning to determine the prevalence of late arterial dilatations at an average of 8.5 years post AAA repair of unruptured aneurysms. Of 94 patients that underwent CT scanning, $64.9 \%$ had aneurysmal dilatation $(>3 \mathrm{~cm})$ and the abnormality was considered significant for surgical repair in $13.8 \%$. 39 patients underwent initial repair with a tube graft of which $12(30.8 \%)$ had iliac aneurysms and $6(15.4 \%)$ of these were considered surgically significant [2]. Although the majority of aneurysmal dilatations were not considered surgically significant, it is evident that late follow-up CT scans are useful in establishing further aneurysmal abnormalities. The authors recommend routine CT scanning at 5 years post AAA repair.

Including true and pseudoaneurysms, there is a wide incidence range of between less than $1 \%$ and up to $30 \%$ at 15 -year post-op review [4-6]. Based in Italy, Locati et al. reviewed 2183 patients with prosthetic grafts inserted over 15 years for both AAA and occlusive disease [7]. 24 of these patients were treated for paraanastomotic aneurysms, at an average interval of 7.7 years post primary graft insertion. Pseudoaneurysms were detected in 19 cases with $47 \%$ proceeding to rupture. CT scanning was performed in all but 4 symptomatic patients whom proceeded directly to theatre. It is not clearly elucidated whether the asymptomatic patients were scanned during routine follow-up or for another reason. The authors suggest the incidence of paraanastomotic aneurysms increases proportionately with postoperative time and as such a screening program would be beneficial in this population.

The literature today distinguishes a link between abdominal aortic aneurysms (AAAs) and femoral and popliteal artery aneurysms. Untreated peripheral aneurysms are limb threatening due to their capability to rupture or cause embolism [8]. Two retrospective studies from the University of Michigan have illustrated a $3.2 \%$ to $3.9 \%$

*Corresponding author: Perera KG, Eastern Health, Nil financial support, 8 Arnold St., Box Hill, Victoria 3128, Australia, Tel: +61403549927; Fax: +613 8396 8988; E-mail: kalpagperera@gmail.com

Received January 29, 2015; Accepted November 09, 2015; Published November 17,2015

Citation: Perera KG, Wong E, Yasir H (2015) Open Abdominal Aortic Aneurysm Repair: Detection of Delayed Aneurysmal Complications. J Vasc Med Surg 3: 228 doi:10.4172/2329-6925.1000228

Copyright: (c) 2015 Perera KG, et al. This is an open-access article distributed under the terms of the Creative Commons Attribution License, which permits unrestricted use, distribution, and reproduction in any medium, provided the original author and source are credited. 
incidence of peripheral aneurysms in patients diagnosed with AAAs $[9,10]$. Diwan et al. conducted a prospective study on 313 consecutive patients with AAAs diagnosed from 1995 to 1998 in order to establish the true incidence of femoral and popliteal artery aneurysms in this population [11]. Of the 313 patients, 36 (12\%) were diagnosed with a total of 51 femoral and popliteal aneurysms; all of which were male. A further 25 patients (8\%) were found to have 26 distinct focal dilatations that were insufficient to meet the aneurysm criteria of the study. As evidenced by their use of Doppler ultrasound, the authors set out to specifically evaluate the incidence of femoral and popliteal artery aneurysms in this population. Patients with peripheral artery pseudoaneurysms, also at risk of rupture if left untreated, were excluded from this study. The study establishes the incidence of peripheral aneurysms, allowing future scope for follow-up of the significance of their findings.

A review of the relevant literature finds mention of metachronous aneurysms following abdominal aortic aneurysm repair, yet there is a scarcity of direct studies investigating this. Evident in the inherent risks of untreated metachronous and paraanastomotic aneurysms, multiple publications invite future study in this area. As such, there is indeed scope for follow-up of patients in our specific population to establish a surveillance program to benefit patient outcomes.

\section{Methods}

Patients having undergone open AAA repair at Eastern Health at least 5 -years prior to the study date were identified. Data was available from the Melbourne Vascular Surgery Audit (MVSA) of a total of 171 procedures performed in between 2003-2008.

Among the 171 patients were 137 males and 34 females, ranging in age from 53 to 92 years (mean age, $74 \pm 8$ ) Of the total cases 81 were referred from outpatients or private rooms, 61 admitted via the emergency department, 23 transferred from other hospitals, 4 referred by another inpatient unit and two cases were not recorded as to source of referral. 65 were emergency operations and 90 cases elective, with a further 16 recorded as semi-urgent.

Due to prospective patient review with radiology, formal ethics approval was gained from the Eastern Health Ethics Committee.

Patient records were investigated to establish mortality and morbidity status of all cases where possible. Those confirmed as deceased or requiring high level nursing care were excluded from the study. All other patients were mailed documentation, inviting participation in the study. Eligible and willing patients were reviewed in clinic and offered CT evaluation where there was no recent scan available.

\section{Results}

171 operations were identified between 2003- 2008. 90 (53\%) patients were confirmed as deceased; with a further $45(26 \%)$ unable to be contacted. A total of 36 responses were received from 93 (54\%) letters sent out inviting patients to participate in the study. 9 patients attended a review clinic, one of whom passed away prior to scanning. The eight patients reviewed were seen between 6-10 years (mean, 8 years) since their surgery. Five (63\%) were found to have developed a para-anastomotic aneurysm and a further 3 (25\%) CIA aneurysms (defined as $>1.8 \mathrm{~cm}$ ) were detected in two patients (Table 1).

The average abdominal aortic aneurysm diameter was $4.0 \pm 1.1 \mathrm{~cm}$ (range, 3.0 to $5.8 \mathrm{~cm}$ ) and the average common iliac artery diameter was $2.3 \pm 0.5 \mathrm{~cm}$ (range, 1.8 to $2.8 \mathrm{~cm}$ ). One patient had a unilateral
CIA aneurysm and another patient had bilateral CIA aneurysms. Of the five cases with para-anastomotic aneurysms, one had a metachronous ascending aortic aneurysm (Table 2).

All patients reviewed with imaging were male, of which all had hypertension and were a current or ex-smoker. Renal impairment and diabetes mellitus were not significant factors in the development of new aneurysms.

\section{Discussion}

Open surgical repair (OSR) has always been the gold standard in AAA management. Yet, with the continual development of endovascular aneurysm repair (EVAR), there is an impending paradigm shift [12]. In the typically comorbid AAA patient, OSR is generally deemed a single definitive procedure, with minimal postoperative follow-up once immediate survival is established. EVAR, with its inherent risks of endoleak, rather mandates a much closer follow-up protocol that involves repeated radiation exposure and renal toxicity with contrast scanning. Standardised follow-up protocols recommend a contrast-enhanced CT scan to be done at 1,6 and 12 months post-operatively and then annually thereafter [13]. Conversely, open surgical repair often mandates only a post-operative review; with the subsequent 5-year scan often not performed.

As shown by our study, 5-year follow-up is often missed and thus secondary complications cannot be addressed. $63 \%$ of cases developing para-anastomotic aneurysms suggest there is indeed a risk of propagating aneurysmal disease following surgical AAA repair. Essentially a precursor to para-anastomotic aneurysm (PAA) formation, a retrospective cohort study by Sonesson et al. found there was a significant dilatation of both the infrarenal aortic neck and the juxtarenal aorta following open aortic procedures [14]. Although small, this tendency indeed reflects the natural history of aneurysmal disease.

The literature suggests PAAA are of greater prevalence amongst ruptured AAA repairs rather than elective procedures [15]. In our study only one case was subsequent to an emergency AAA repair, yet with our small sample size, this is not representative of the greater population.

Scientific data suggests clear genetic and epidemiological associations between an aneurysmal aorta and peripheral aneurysmal disease [16]. As such a common pathological basis for the development of metachronous aneurysms may be inferred. An exploratory study performed in the Netherlands noted a significant correspondence in proinflammatory transcription factors and activation proteases between AAA and peripheral arterial aneurysms [17].

Open repair has historically been the favoured approach in managing aortic aneurysms in patients aged 65 years and younger.

\begin{tabular}{|c|c|}
\hline Characteristic & Study $\mathbf{( N = 8 )}$ \\
\hline Female & 0 \\
\hline Male & $8(100 \%)$ \\
\hline Diabetes mellitis & $1(12.5 \%)$ \\
\hline Renal impairment (eGFR<50) & $2(25 \%)$ \\
\hline Hypertension & $8(100 \%)$ \\
\hline Ischaemic Heart Disease & $5(62.5 \%)$ \\
\hline Hypercholesterolemia & $7(87.5 \%)$ \\
\hline Smoking (ex or current) & $8(100 \%)$ \\
\hline Airways disease & $4(50 \%)$ \\
\hline
\end{tabular}

Table 1: Characteristic studies of different diseases. 


\begin{tabular}{|c|c|c|c|c|c|c|c|c|c|c|c|}
\hline \multirow{2}{*}{ Demographic } & \multirow{2}{*}{ EM / EL } & \multirow{2}{*}{ D.O.S } & \multirow{2}{*}{ Post-op (Years) } & \multicolumn{7}{|c|}{ Co-morbidities } & \multirow{2}{*}{ Outcome } \\
\hline & & & & DM & eGFR & Resp & $\mathrm{Htn}$ & IHD & Smoker & Chol & \\
\hline HB 84M & Elective & $12 / 2007$ & 6 & $\mathrm{~N}$ & 42 & COAD & $\mathrm{Y}$ & $\mathrm{Y}$ & Ex & $\mathrm{Y}$ & $3.7 \mathrm{~cm}$ AAA \\
\hline DG 80M & Elective & $12 / 2005$ & 8 & $\mathrm{~N}$ & 73 & COAD & $Y$ & $N$ & Ex & Y & $\begin{array}{c}5.8 \mathrm{~cm} \text { AAA } \\
2.2 \mathrm{~cm} \mathrm{~L} \mathrm{CIAA}\end{array}$ \\
\hline NB 78M & Elective & $09 / 2004$ & 9 & $\mathrm{~N}$ & 59 & Asthma & $Y$ & $Y$ & Ex & $\mathrm{Y}$ & Nil aneurysm \\
\hline JF 69M & Elective & $10 / 2003$ & 10 & Y & 59 & $N$ & $Y$ & $N$ & Ex & $N$ & $3.0 \mathrm{~cm}$ AAA \\
\hline MP 65M & Elective & $11 / 2005$ & 8 & $\mathrm{~N}$ & 90 & $\mathrm{~N}$ & $\mathrm{Y}$ & $\mathrm{Y}$ & Ex & $\mathrm{Y}$ & $\begin{array}{l}2.8 \mathrm{~cm} \text { R CIAA } \\
1.8 \mathrm{~cm} \mathrm{~L} \mathrm{CIAA}\end{array}$ \\
\hline RJ 80M & Emergency & $04 / 2005$ & 8 & $\mathrm{~N}$ & 33 & COAD & $Y$ & $Y$ & Y & Y & $4.4 \mathrm{~cm} \mathrm{AAA}$ \\
\hline GP 76M & Elective & $12 / 2005$ & 8 & $\mathrm{~N}$ & 55 & $\mathrm{~N}$ & $Y$ & $Y$ & Ex & Y & Nil aneurysm \\
\hline JS 80M & Elective & $12 / 2006$ & 7 & $\mathrm{~N}$ & 53 & $\mathrm{~N}$ & $\mathrm{Y}$ & $\mathrm{N}$ & Ex & Y & $3.3 \mathrm{~cm}$ AAA \\
\hline
\end{tabular}

With uncertainties regarding long-term graft durability, risk of late graft rupture and surveillance associated radiation exposure; endovascular repair is generally discouraged in younger patients [1820]. Nonetheless, a single-centre study on elective AAA repairs by Altaf et al. found similar reintervention rates in open (13\%) compared to endovascular $(11 \%)$ procedures at a median follow-up interval of 77 months [20]. On the contrary, a retrospective review of ruptured aneurysms in another United States hospital noted a much higher re-intervention rate following EVAR than open repair $(10.9 \%$ vs $1.5 \%)$ [21]. It is worth noting that this study population was solely on ruptured aneurysms, with a higher average age at initial intervention and median follow-up interval of only 36 months.

Despite 171 procedures having been completed during our study period; ultimately reviewing a mere 9 patients is quite a poor return. 93 letters were sent out with a total of 36 (39\%) responses. Indeed, the morbidity and mortality associated with both the disease and procedure lends to an inherently poor response rate amongst this population. Yet, a health related survey sent out to patients up to 8.5 years post-ruptured AAA repair in Quebec, Canada had a $75 \%$ completion rate [22]. Further, Khan et al. had an $89 \%$ response rate in their questionnaire establishing patients' perspective of functional outcome following elective AAA repair [23]. Nonetheless, divergent to our correspondence these two studies utilised simple surveys a patient could complete at their own leisure. As our study required patient's to physically attend up to two clinic appointments with the likelihood of a repeat $\mathrm{CT}$ scan, voluntary inclusion indeed presents a more arduous endeavour than a mere survey. Future studies may potentially be streamlined by scheduling a scan, when required, on the same day as the clinic appointment.

Of the nine patients reviewed in our study, 2 cases (22\%) developed CIAAs; one unilateral and one bilateral. With a range from 1.8-2.2 $\mathrm{cm}$, all of these were asymptomatic and only detected with our repeat imaging. Iliac artery aneurysms are noted to be the most common aneurysm after AAA, with CIAA the most frequent of these [24]. The majority of iliac artery aneurysms develop in patients with AAA. A CT angiographic study from the UK suggests $20 \%$ of patients with AAA have CIAA [25]. Rather, with an incidence of $0.03-2 \%$ in autopsy series', isolated iliac artery aneurysms are exceeding uncommon [2628]. A series published by Huang et al. found $86 \%$ of patients with CIAA having existing AAA and 65\% with contralateral CIAA [29]. Thus, it indeed seems prudent to schedule follow-up imaging post AAA repair to monitor for peripheral arterial aneurysms.

Common practice regarding threshold for repair is $3 \mathrm{~cm}$ due to likelihood of becoming symptomatic and increased risk of rupture [27, 30-32]. Similar to AAA, ruptured CIAA carries high mortality rates. Further, concurrent AAA rupture will indeed complicate management.
As with AAA surgery, open repair has long been the gold standard for management of iliac aneurysms until the recent development of an endoluminal approach [33-37]. Patel et al. found no difference in primary patency rates between open and endovascular repair of isolated iliac artery aneurysms over 12 years [38]. With a median follow-up of 36 months, Fossaseca et al. describe primary and secondary patency rates of $95 \%$ and $100 \%$ respectively of endovascularly treated iliac aneurysms [39]. Similarly, Boules et al. note a primary patency rate of $95 \%$ at 2 years post endovascular repair of iliac artery aneurysms [40]. Thus, endoluminal repair provides a safe, minimally invasive option for CIAA repair with favourable anatomy without compressive symptoms.

We acknowledge that the study is inherently limited by a significant loss to follow-up and therefore small sample size. Future studies may involve longer, prospective follow-up practices and could consider investigating rates of subsequent rupture and reintervention.

\section{Conclusion}

Despite our small sample size, we found a high proportion of paraanastomotic and metachronous aneurysms following open AAA repair. Indeed, our study results suggest that closer radiological monitoring be considered for open AAA repair. Not as frequent as per EVAR, yet we suggest that at least a 5 -year post-operative scan be completed. Further, with studies showing similar re-intervention rates in the elective setting between the two approaches, it may perhaps be wise to consider EVAR as the primary intervention in the younger patient.

\section{References}

1. Jeffrey JM, Robert M, Thompson W (2012) Clinical evaluation of abdominal aortic aneurysm. In UpToDate Inc.

2. Kalman P, Kalman (1999) The value of late computed tomographic scanning in identification of vascular abnormalities after abdominal aortic aneurysm repair Journal of vascular surgery 29: 442-450.

3. Hallett JW Jr, Marshall DM, Petterson TM, Gray DT, Bower TC, et al. (1997) Graft-related complications after abdominal aortic aneurysm repair: reassurance from a 36 -year population-based experience. Journal of vascular surgery 25: 277-284

4. Edwards JM, Teefey SA, Zierler RE, Kohler TR (1992) Intraabdomina paraanastomotic aneurysms after aortic bypass grafting. Journal of vascular surgery 15: 344-350.

5. Millili JJ, Lanes JS, Nemir P Jr (1980) A study of anastomotic aneurysms following aortofemoral prosthetic bypass. Ann Surg 192: 69-73.

6. Szilagyi DE, Smith RF, Elliott JP, Hageman JH, Dall'Olmo CA (1975) Anastomotic aneurysms after vascular reconstruction: problems of incidence, etiology, and treatment. Surgery 78: 800-816.

7. Locati P, Socrate AM, Costantini E (2000) Paraanastomotic aneurysms of the abdominal aorta: a 15-year experience review. Cardiovasc Surg 8: 274-279.

8. Whitehouse WM Jr, Wakefield TW, Graham LM, Kazmers A, Zelenock GB 
Citation: Perera KG, Wong E, Yasir H (2015) Open Abdominal Aortic Aneurysm Repair: Detection of Delayed Aneurysmal Complications. J Vasc Med Surg 3: 228. doi:10.4172/2329-6925.1000228

et al. (1983) Limb-threatening potential of arteriosclerotic popliteal artery aneurysms. Surgery 93: 694-699.

9. Dent TL, Lindenauer SM, Ernst CB, Fry WJ (1972) Multiple arteriosclerotic arterial aneurysms. Arch Surg 105: 338-344.

10. Graham LM, Zelenock GB, Whitehouse WM Jr, Erlandson EE, Dent TL, et al. (1980) Clinical significance of arteriosclerotic femoral artery aneurysms. Archives of surgery 115: 502-507.

11. Diwan A, Sarkar R, Stanley JC, Zelenock GB, Wakefield TW (2000) Incidence of femoral and popliteal artery aneurysms in patients with abdominal aortic aneurysms. J Vasc Surg 31: 863-869.

12. Hertzer NR, Mascha EJ, Karafa MT, O'Hara PJ, Krajewski LP, et al. (2002) Open infrarenal abdominal aortic aneurysm repair: the Cleveland Clinic experience from 1989 to 1998 . Journal of vascular surgery 35: 1145-1154.

13. Walker TG, Kalva SP, Yeddula K, Wicky S, Kundu S, et al. (2010) Clinical practice guidelines for endovascular abdominal aortic aneurysm repair: written by the Standards of Practice Committee for the Society of Interventional Radiology and endorsed by the Cardiovascular and Interventional Radiological Society of Europe and the Canadian Interventional Radiology Association. J Vasc Interv Radiol 21: 1632-1655

14. Sonesson B, Resch T, Länne T, Ivancev K (1998) The fate of the infrarenal aortic neck after open aneurysm surgery. J Vasc Surg 28: 889-894.

15. Cho JS, Gloviczki P, Martelli E, Harmsen WS, Landis ME, et al. (1998) Longterm survival and late complications after repair of ruptured abdominal aortic aneurysms. J Vasc Surg 27: 813-819.

16. Jacob T, Hingorani A, Ascher E (2001) Examination of the Apoptotic Pathway and Proteolysis in the Pathogenesis of Popliteal Artery Aneurysms. European Journal of Vascular and Endovascular Surgery 22: 77-85.

17. Abdul-Hussien H, Hanemaaijer R, Kleemann R, Verhaaren BF, van Bockel $\mathrm{JH}$, et al. (2010) The pathophysiology of abdominal aortic aneurysm growth: corresponding and discordant inflammatory and proteolytic processes in abdominal aortic and popliteal artery aneurysms. Journal of vascular surgery 51: $1479-1487$

18. van Beek SC, Conijn AP, Koelemay MJ, Balm R (2014) Editor's Choice Endovascular Aneurysm Repair Versus Open Repair for Patients with a Ruptured Abdominal Aortic Aneurysm: A Systematic Review and Meta-analysis of Short-term Survival. European Journal of Vascular and Endovascular Surgery 47: 593-602.

19. Dangas G, O'Connor D, Firwana B, Brar S, Ellozy S, et al. (2012) Open versus endovascular stent graft repair of abdominal aortic aneurysms: a meta-analysis of randomized trials. JACC Cardiovasc Interv 5: 1071-1080.

20. Altaf N, Abisi S, Yong Y, Saunders JH, Braithwaite BD, et al. (2013) Midterm results of endovascular aortic aneurysm repair in the young. Eur $\mathrm{J}$ Vasc Endovasc Surg 46: 315-319.

21. Edwards ST, Schermerhorn ML, O'Malley AJ, Bensley RP, Hurks R, et al. (2014) Comparative effectiveness of endovascular versus open repair of ruptured abdominal aortic aneurysm in the Medicare population. Journal of Vascular Surgery 59: 575-582.

22. Hill AB, Palerme LP, Brandys T, Lewis R, Steinmetz OK (2007) Health-related quality of life in survivors of open ruptured abdominal aortic aneurysm repair: a matched, controlled cohort study. J Vasc Surg 46: 223-229.
23. Khan JA, Mazari FA, Abdul Rahman MN, Mockford K, Chetter IC, et al. (2011) Patients' perspective of functional outcome after elective abdominal aortic aneurysm repair: a questionnaire survey. Ann Vasc Surg 25: 878-886.

24. McCready RA, Pairolero PC, Gilmore JC, Kazmier FJ, Cherry KJ Jr, et al. (1983) Isolated iliac artery aneurysms. Surgery 93: 688-693.

25. Armon MP, Wenham PW, Whitaker SC, Gregson RH, Hopkinson BR (1998) Common iliac artery aneurysms in patients with abdominal aortic aneurysms. European Journal of Vascular and Endovascular Surgery. 15: 255-257.

26. Brunkwall J, Hauksson H, Bengtsson H, Bergqvist D, Takolander R, et al. (1989) Solitary aneurysms of the iliac arterial system: an estimate of their frequency of occurrence. J Vasc Surg 10: 381-384.

27. Richardson JW, Greenfield LJ (1988) Natural history and management of iliac aneurysms. J Vasc Surg 8: 165-171.

28. Garland HG (1932) The pathology of aneurysm: A review of 167 autopsies. The Journal of Pathology and Bacteriology 35: 333-350.

29. Huang Y, Gloviczki P, Duncan AA, Kalra M, Hoskin TL, et al. (2008) Common iliac artery aneurysm: expansion rate and results of open surgical and endovascular repair. J Vasc Surg 47: 1203-1210.

30. Santilli SM, Wernsing SE, Lee ES (2000) Expansion rates and outcomes for iliac artery aneurysms. J Vasc Surg 31: 114-121.

31. Sacks NP, Huddy SP, Wegner T, Giddings AE (1992) Management of solitary iliac aneurysms. J Cardiovasc Surg (Torino) 33: 679-683.

32. Krupski WC, Selzman CH, Floridia R, Strecker PK, Nehler MR, et al. (1998) Contemporary management of isolated iliac aneurysms. J Vasc Surg 28: 1-11.

33. Marin ML, Veith FJ, Lyon RT, Cynamon J, Sanchez LA (1995) Transfemora endovascular repair of iliac artery aneurysms. Am J Surg 170: 179-182.

34. Minato N, Itoh T, Natsuaki M, Nakayama Y, Yamamoto H (1994) Isolated iliac artery aneurysm and its management. Cardiovasc Surg 2: 489-494.

35. Pitoulias GA, Donas KP, Schulte S, Horsch S, Papadimitriou DK (2007) Isolated iliac artery aneurysms: endovascular versus open elective repair. $J$ Vasc Surg 46: 648-654.

36. Rehring TF, Brewster DC, Cambria RP, Kaufman JA, Geller SC, et al. (2000) Utility and reliability of endovascular aortouniiliac with femorofemoral crossover graft for aortoiliac aneurysmal disease. J Vasc Surg 31: 1135-1141.

37. Scheinert D, Schröder M, Steinkamp H, Ludwig J, Biamino G (2000) Treatment of iliac artery aneurysms by percutaneous implantation of stent grafts. Circulation 102: III253-258.

38. Patel NV, Long GW, Cheema ZF, Rimar K, Brown OW, et al. (2009) Open vs. endovascular repair of isolated iliac artery aneurysms: A 12-year experience. $J$ Vasc Surg 49: 1147-1153.

39. Fossaceca R, Guzzardi G, Di Terlizzi M, Divenuto I, Cerini P, et al. (2013) Long-term efficacy of endovascular treatment of isolated iliac artery aneurysms. Radiol Med 118: 62-73.

40. Boules TN, Selzer F, Stanziale SF, Chomic A, Marone LK, et al. (2006) Endovascular management of isolated iliac artery aneurysms. J Vasc Surg 44: 29-37. 